\title{
A Debris Clearance Robot for Extreme Environments
}

DOI:

10.1007/978-3-030-23807-0_13

\section{Document Version}

Accepted author manuscript

Link to publication record in Manchester Research Explorer

\section{Citation for published version (APA):}

West, C., Arvin, F., Cheah, W., West, A., Watson, S., Giuliani, M., \& Lennox, B. (2019). A Debris Clearance Robot for Extreme Environments. In Towards Autonomous Robotic Systems https://doi.org/10.1007/978-3-030-23807$0 \_13$

\section{Published in:}

Towards Autonomous Robotic Systems

\section{Citing this paper}

Please note that where the full-text provided on Manchester Research Explorer is the Author Accepted Manuscript or Proof version this may differ from the final Published version. If citing, it is advised that you check and use the publisher's definitive version.

\section{General rights}

Copyright and moral rights for the publications made accessible in the Research Explorer are retained by the authors and/or other copyright owners and it is a condition of accessing publications that users recognise and abide by the legal requirements associated with these rights.

\section{Takedown policy}

If you believe that this document breaches copyright please refer to the University of Manchester's Takedown Procedures [http://man.ac.uk/04Y6Bo] or contact uml.scholarlycommunications@manchester.ac.uk providing relevant details, so we can investigate your claim.

\section{OPEN ACCESS}




\title{
A Debris Clearance Robot for Extreme Environments ${ }^{\star}$
}

\author{
Craig West ${ }^{1}$, Farshad Arvin ${ }^{2}$, Wei Cheah ${ }^{2}$, Andrew West $^{2}$, Simon Watson ${ }^{2}$, \\ Manuel Giuliani ${ }^{1}$, and Barry Lennox ${ }^{2}$ \\ 1 Bristol Robotics Laboratory, University of the West of England, Bristol, UK \\ craig.west@uwe.ac.uk \\ 2 Robotics for Extreme Environments Lab at the School of Electrical \& Electronic \\ Engineering, The University of Manchester, UK \\ barry.lennox@manchester.ac.uk
}

\begin{abstract}
The need for nuclear decommissioning is increasing globally, as power stations and other facilities utilising nuclear reaches the end of their operational life. Currently the majority of decommissioning tasks are carried out by workers in protective air fed suits, which is slow, expensive and dangerous. The work presented here is the early stages in the development of a flexible mobile manipulator platform, combining a Clearpath Husky, a Universal UR5 manipulator and various sensors. The system will be used for research specifically in the area of exploration of contaminated environments, map building to aid in task planning, and also to investigate manipulation for waste sorting. The aim is to develop a system that can, in the short term, be used in real world tasks but longer term function as a research platform to allow continued research and development. As well as developing a hardware platform, a detailed simulation model is also being developed to allow testing of algorithms in simulation before being deployed on hardware. The use of the simulation model for operator training is also an area that will be investigated in the future. This article focuses on the planned work for developing the system, as well as discussing the progress on the simulation model.
\end{abstract}

Keywords: Exploration · Autonomous · Extreme Environments · Nuclear · Simulation · Mobile Robots.

\section{Introduction}

Nuclear decommissioning often involves working in areas contaminated with high levels of radiation, where human entry is only possible with the use of protective air fed suits. The use of air fed suits require a team of people to put on and take off, and following the use of the suit it becomes contaminated waste which needs to be disposed of accordingly. Besides being costly and time consuming,

\footnotetext{
* This work was supported by EPSRC RNE (No. EP/P01366X/1) project and UK Research and Innovation via the TORONE consortium (EP/P018505/1). The second author would like to thank EPSRC Impact Acceleration Account (EP/R511626/1).
} 
the working time for the operators wearing these protective suits are limited due to the radiation risk [1]. These are contributing factors to why nuclear decommissioning is expensive, slow and dangerous. Nonetheless, nuclear decommissioning remains a critical task that needs to be undertaken.

The need for decommissioning is in fact increasing as more facilities are approaching the end of their operational life. One such example is the Sellafield site in the UK, where the Thermal Oxide Reprocessing Plan (THORP) has been recently shut down, and the Magnox reprocessing plant is due for closure before 2020 [10]. These facilities potentially contain a variety of contaminated materials.

It is required that the contamination level of items in these areas is confidently identified so that the items can be processed and sorted accordingly. There are four levels of radiation contamination, High level, Intermediate level, low level, very low level [18] and each level requires different handling. For example very low level waste can be disposed of with normal rubbish, but low level waste needs to be stored in special near surface or below surface facilities. Therefore misclasifying waste has a significant impact on costs, also if the contamination level can not be confidently determined it has to be treated as higher level further impacting costs and time scales.

With this in mind, this work aims to develop a mobile manipulator platform for remote operation in these hazardous areas. By combining a mobile base with a manipulator, namely a Clearpath Husky robot [3] with a Universal Robots UR5 robot arm [17], a platform that can operate remotely, indoors, and perform a variety of useful tasks will be created.

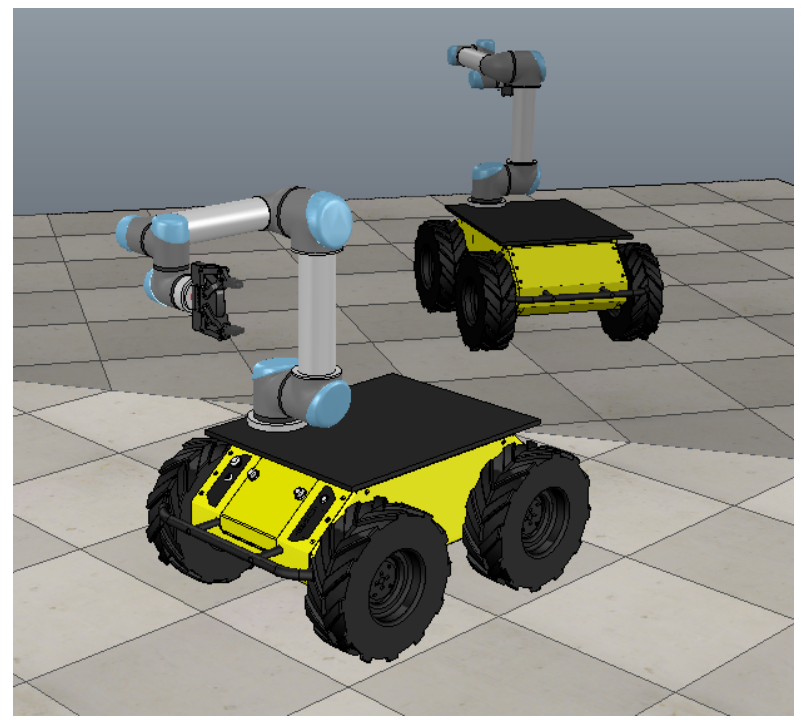

Fig. 1. V-REP simulation of a Clearpath Husky robot with a UR5 robot arm mounted to it. 


\subsection{Robot Platform}

The Husky was chosen for being a good compromise between its small size and maneuverability for operating in confined or cluttered spaces, whilst still large enough to mount a manipulator and other sensors. For example, its small size allows it to traverse through doorways, under tables, and also over small obstacles or uneven terrain that may be encountered in a nuclear facility. The Universal UR5 arm is compact enough to be mounted on the Husky (see fig. 1) and has a payload of $5 \mathrm{~kg}$, which is useful for real-world manipulation tasks. The manipulator is also widely supported manipulator and is available off-the-shelf in an integrated package with the Husky.

Applications for this system will range from visual inspection and mapping, to manipulation and waste sorting. This will give operators a view inside these hazardous facilities, some of which have not been entered for many years, allowing necessary tasks to be identified and a decommissioning plan to be constructed. The same system can be used for task such as moving and sorting waste material, or collecting samples. There is also the potential to fit specialist tools as the manipulator's end-effector, such as a saw for cutting large waste items to downsize it for storage. Although this has potential to cause other issues, such as creating contaminated dust, so is an area that would require further consideration.

\subsection{Challenges}

The challenges presented by these environments extend beyond the obvious radiation risks, and include:

- chemical hazards

- operating in confined spaces

- operating in potentially unknown environments as some facilities have been closed for many years

- communication challenges presented by thick steel re-enforced concrete walls

- materials may have perished over time for example metal rusting which may affect the handling of objects.

The dangers posed by these hazards can be minimized by the use of robotic systems instead as the environment is dangerous, unknown and potentially unpredictable. Even so, these environments do pose a challenge for robotic systems. Communication between robot and user is of utmost importance in the target deployment scenario. However, the work environment results in unreliable wireless communication. Hence, a tether is proposed in this work to allow communication between the operator and the robot. This also gives the advantage of extending operation time beyond the limited on-board battery. As such, part of this work is to develop a tether management system that will consider the tether during path planning and aim to avoid problems other tethered robots have faced. This is discussed below.

Another challenge that has to be considered with the deployment of robots in radiation contaminated environment is what happens to the robot post operation, or if it breaks. The robot will have become radiologically contaminated 
and thus requires a complete radiation cleanup which is very difficult and often deemed not worth the time and cost. More commonly, contaminated systems are disposed of which in turn creates more contaminated waste that needs to be disposed off. This forms part of the motivation for developing a flexible system that can perform a variety of tasks rather than a highly specialised robot for one particular task. A system that can operate for a prolonged period and perform a range of tasks could be very beneficial to the decommissioning industry.

With these considerations in mind, we chose to make use of proven off-theshelf components to create the mobile manipulator platform, rather than an entirely bespoke solution. This approach will yield a reliable system which is of high importance for the target application. The system will also be modular, allowing parts such as sensors to be easily replaced if they get damaged. Although the replacement of parts requires human intervention, this approach is able to extend the lifetime of the system, reducing both cost and further contaminated waste.

\section{Background}

The use of mobile robots in the nuclear industry is not a new idea, [16] gives a detailed review of mobile robots deployed in the nuclear industry over the last few decades. Many robots used previously have been used in response to accidents such as at Chernobyl or Fukushima, with relatively few robots being used in decommissioning tasks. A similar platform to the one discussed here has also been developed by the Nuclear and Applied Robotics Group at the University of Texas at Austin, called Vaultbot [14]. Their system combines a Husky with dual UR5 manipulators. However their system required extensive modification of the Husky platform to allow two manipulators to be fitted, and being battery powered it has a short run time. In contrast our system requires almost no modification and so is kept modular and retains the mean time between failure (MTBF) of the off-the-shelf hardware. It will also utilise a tether with a novel tether management system, allowing extended periods of operation and maintain communication in environments where wireless communication is unreliable.

The main contributions of this work come from the combination of expertise from different UK Institutions to create:

- A reliable mobile platform with semi-autonomous grasping abilities

- A tether management system to prevent cable tangling with obstacles

- An intuitive user interface that is effective and simple to use, shared by the real hardware and simulation model

- An accurate simulation model, which somewhat uniquely, includes the tether in the model

The platform will be developed focusing on real world challenges but will also allow novel research into areas such as grasping, manipulation and human robot interfaces. The aim is to develop a system that in the short term can be deployed in the real world, and with minimal operator training be used by 
nuclear industry workers, whilst simultaneously in the longer term be used as a research platform to develop industry relevant functionality. Another benefit of using off-the-shelf components is that the platform can be replicated at each of the partner institutions, allowing research in different aspects to be carried out in parallel to each other.

As such the first iteration will use a direct teleoperation system for both the mobile base and the manipulator. This will allow the basic system to be assembled with the various sensors integrated and tested. Once the basic system is working other areas can be investigated such as semi-autonomous algorithms for both the mobile base and the manipulator as well as different user interfaces.

There is also scope to use the platform for further research into, for example, human factors experiments, studying the effects of different sensors on ease of tele-operation, or different input methods and levels of automation. This could involve carrying out a series of tests with different cameras attached, e.g. a fixed camera, fixed stereo cameras, pan-tilt camera system, fish eye or 360 camera, and also comparing between on screen images with the use of a VR headset. To investigate different input methods and levels of automation, a study comparing e.g. keyboard tele-operation, game pad control and point and click interactive target markers could also be carried out. These two areas overlap, as the control method has a direct impact on the information required by the operator.

A reliable communication link between a remote inspection robot and operators is invaluable. In nuclear environments, the thick concrete walls designed to shield against ionising radiation attenuate most radio signals as to become unusable, thus making wireless communications impractical in most cases.

A wired connection between the robot and operators offers a reliable and usable solution to the problem of data transfer. The major drawback is the additional weight, mechanical complexity, and the threat of a cable tether becoming tangled or caught on obstacles, incapacitating the robot. The latter ended remote robotic inspection operations of Unit 2 at the Fukushima Daiichi Nuclear Power Plant. The tether management system became jammed, immobilising the Quince 1 robot [9], resulting in loss of communications and the robot had to be abandoned [20]. Tether management for this robot was sited as the most significant problem faced for inspection of Unit 2 and 3.

Mechanical considerations can be made to try minimise tangling events, but robot path planning can play an important role in avoiding tether crossing and minimising cable length. Previous work on path planning of single tethered robots have concentrated on minimising cable length for a given goal position, with the tether fixed to a base position [19].

The majority of work on single tethered robot path planning has allowed for the robot to cross its tether if it would result in the shortest path (so-called semi-planar, compared to purely planar where no crossing occurs) $[2,7,8,13,19]$. In real-world robotic deployment scenarios crossing of a communications cable could lead to wear and damage of the tether, as well as risk of tangling.

It is common for robots to utilise an occupancy grid [15] for obstacle mapping and path planning due to the ubiquity of the Robot Operating System (ROS) 
[12] and its native navigation stack [6]. The solutions proposed in previous works require absolute knowledge of all obstacles, and the ability to describe them as a polygon with a centre [7]. As obstacles are typically identified as locations where an instrument such as lidar or depth camera has encountered an object, then marked on a costmap, it is not readily possible to fully identify and describe every distinct obstacle as required by these algorithms.

Existing algorithms further require full knowledge of an obstacle map to be able to compute possible paths, ultimately making requests for path planning quicker. This pre-computation can take tens of minutes [8], but in an unknown environment, an obstacle map may be updated on the order of every few seconds. Computation time of an hour before being able to make a path planning request is infeasible for every time the map is updated. Finally, these algorithms minimise path length of the tether, a consequence is that the tether comes into direct contact with obstacles, under the assumption the cable can be pulled taut. This acts to minimise cable length, however, in unstructured and cluttered nuclear environments, it would be an unacceptable risk to possibly move or topple an obstacle which may contain contaminated waste.

A path planner for a single tethered robot for unstructured nuclear environments would need to avoid direct contact of obstacles with the tether, forbid crossing of the tether at the expense of time or cable length, be able to adapt to changes in the obstacle map with minimal computational overhead, and cope with a gridded costmap representation of obstacles in the environment.

To test such a path planner, it is advantageous to use a robot simulation which can mimic not only robot motion, but most importantly the dynamics of the tether.

\section{Simulation}

Whilst the hardware is purchased and assembled to create the mobile manipulator, a simulation model has been created using the V-REP simulation software [4]. V-REP was chosen based on the recommendations of previous work by Pitonakova et al. [11], which compared the performance of different simulation environments. The Clearpath Husky model is not included in V-REP, however a CAD file of the complete Husky is available from Clearpath, and so this was used as a base to create the assembly for the simulation model. The finished model has a UR5 manipulator, which is readily available in V-REP, attached as shown in fig. 1. Due to the detail in this model, particularly the geometry of the tyres, the physics based simulation runtime was very slow, and so a simplified version was also created as shown in fig. 2. While this version has simplified geometry, properties such as mass and friction are maintained so the dynamic behaviour of the real system is still represented closely.

Both of these models can be integrated through V-REP with ROS, eliminating code porting issues between the real and simulated robot. This allows new algorithms to be quickly and safely developed in simulation before being deployed on the hardware. It also allows the possibility of operator training on 
a simulated robot, using the same control interface, before using the hardware. This could be particularly useful where following an exploration and mapping stage, the real environment can be simulated, allowing navigation or manipulation tasks to be evaluated prior to executing the same tasks in the real world.

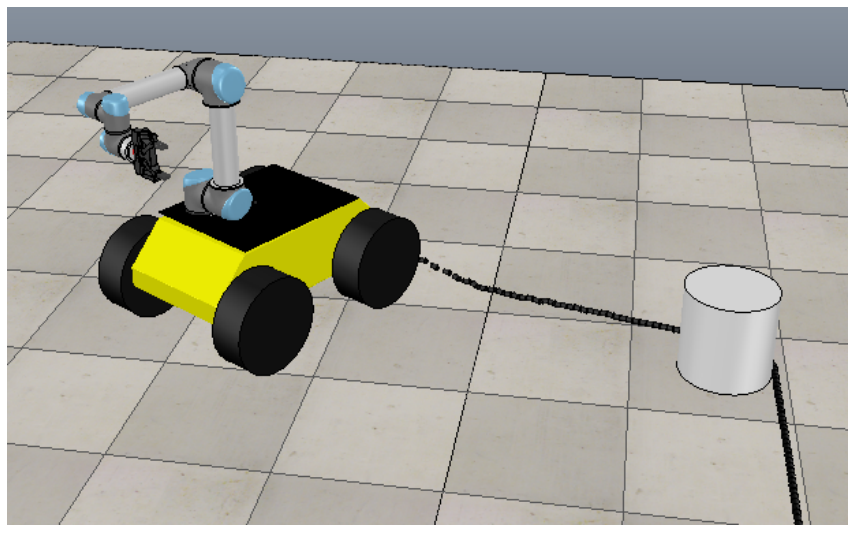

Fig. 2. Simplified model of Clearpath Husky in V-REP with UR5 mounted and power/communication tether attached.

\subsection{Tether Simulation}

As part of the simulation, a tether has also been created, shown in fig. 2 (note that the tether can be added to the detailed model also). Again, V-REP does not include cables in the model library hence it has been developed from the ground up. The tether was created by forming a kinematic chain of small cylinders connected by joints. Each cylinder has physical properties such as mass and friction and each joint, as well as providing flexibility, acts as spring damper system. Tuning these parameters allowed realistic cable behaviour to be achieved as a result of the physics engine in V-REP, as illustrated in fig. 3 where the cable can be seen sagging either side of the solid block and being pulled onto the motorized drum.

The tether is fixed to the rear of the platform and having both mass and friction acts realistically as it is dragged along the floor. To the authors current knowledge there are no examples of a tether being included in a mobile robot simulation, despite the impact it has on the path planning and the effect on dynamic properties from dragging a cable along the floor that can snag or interact with obstacles. Including the tether in the simulation also allows the tether management solution to be evaluated as it is developed, facilitating rapid and safe development. 


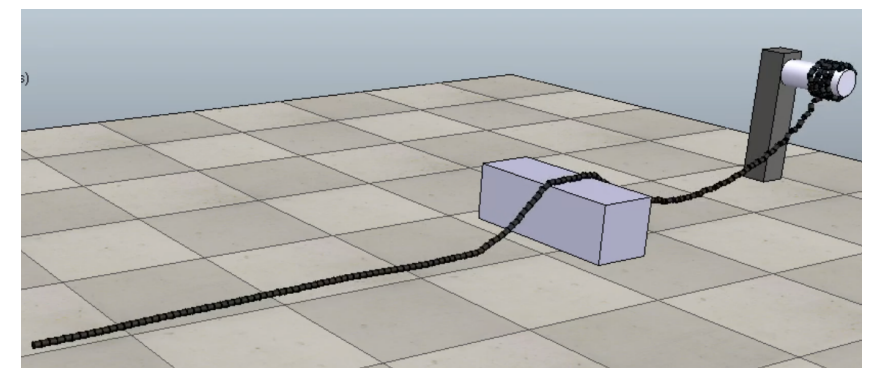

Fig. 3. Example from testing the cable, cable is wound onto a motorized drum whilst being dragged over block.

\subsection{Hardware Configuration}

During the development of the simulation model it became apparent that the placement of the manipulator has a noticeable effect on the performance of the system. Results of an initial investigation placing the manipulator at the front, middle and rear of the simulated Husky and monitoring the XY position of the robot when given identical motor inputs are shown in fig. 4. This is an area that needs further investigation as the difference seems much larger than would be expected. It is assumed that the difference is caused by the change in the robot's centre of mass affecting traction. Results such as these show one of the benefits of simulation, these effects can be easily investigated by changing the robot configuration, which would be a significant undertaking with the real hardware.

To determine whether this difference is exaggerated in the simulation a test using the real Husky will be carried out when the hardware is available. The results shown here are with the manipulator static, so the manipulator moving whilst the base is moving would potentially have a large effect on performance. This may have an impact on the control of the system when objects are being carried, for example it may be required that the manipulator remains static whilst the base is moving, as such understanding if this is as noticeable on the real robot is important. On the other hand, using a SLAM algorithm and semi autonomous navigation may provide enough performance to mitigate the effect. Testing on the real hardware is necessary to better understand the issue and will also help to improve the simulation model, narrowing the reality gap.

Another area that is currently being investigated is what sensors are needed on the robot. It is desirable to keep the system as simple as possible whilst also making it capable enough to be easily controllable and with some semiautonomous behaviours. It is anticipated that at a minimum a 2D Lidar, a camera and a radiation sensor will be needed. The 2D Lidar will allow SLAM (Simultaneous localisation and mapping) so that the environment can be navigated. A camera on the Husky will allow the operator to see what the robot can see, this is essential for tele-operation. Additionally using stereo or depth cameras would allow a 3D point cloud of the environment to be created which would aid planning of tasks and visualizing the area. It is also likely a second 


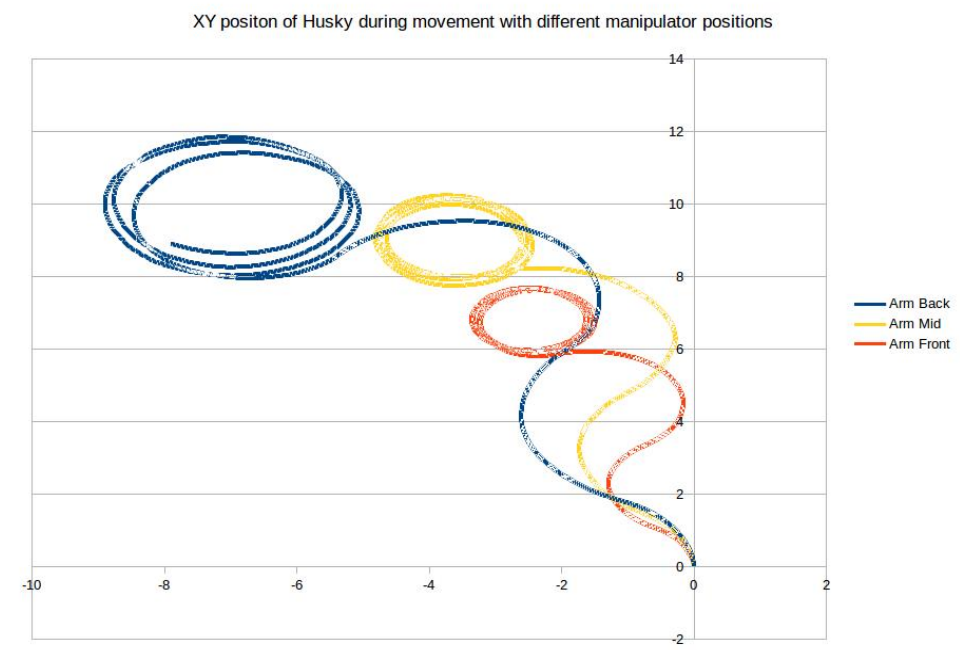

Fig. 4. Initial results showing XY position of the platform, with the same motor input, for 3 different manipulator mounting locations.

camera would be needed on the manipulator, to act as an eye in hand camera to allow grasping. A radiation sensor would allow both monitoring of the environment to identify radiation level and also aid in separating items into high and low level waste. Date from the radiation sensor could potentially be combined with a $3 \mathrm{D}$ point cloud, to give a $3 \mathrm{D}$ environment map showing areas of contamination. Having the detailed simulation model allows different configurations and positioning of sensors to be tested, this will give a greater level of confidence when assembling the hardware as we can test that one sensor placement doesn't occlude another and that all the desired sensors fit on the platform for example.

\subsection{User Interface}

The simulation model being integrated with ROS allows different combinations of sensors to be tested in simulated environment and the output to be displayed in RVIZ as it would be with real hardware sensors. This allows quickly testing different sensor combinations and seeing the output on the user interface before implementing with expensive hardware. An example of this is shown in fig. 5, which shows the combined output of a simulated 2D Lidar and a low resolution depth camera $(64 \times 64$ pixel) in a simulated corridor. The 2D Lidar data is used with a SLAM algorithm, currently GMapping [5], to produce a 2D map of the area and the depth camera creates a $3 \mathrm{D}$ point cloud. The investigation into different sensor combinations is ongoing, and more work is required to identify suitable sensors. A user study may be carried out to identify what data the operators want which can then help identify necessary sensors. 


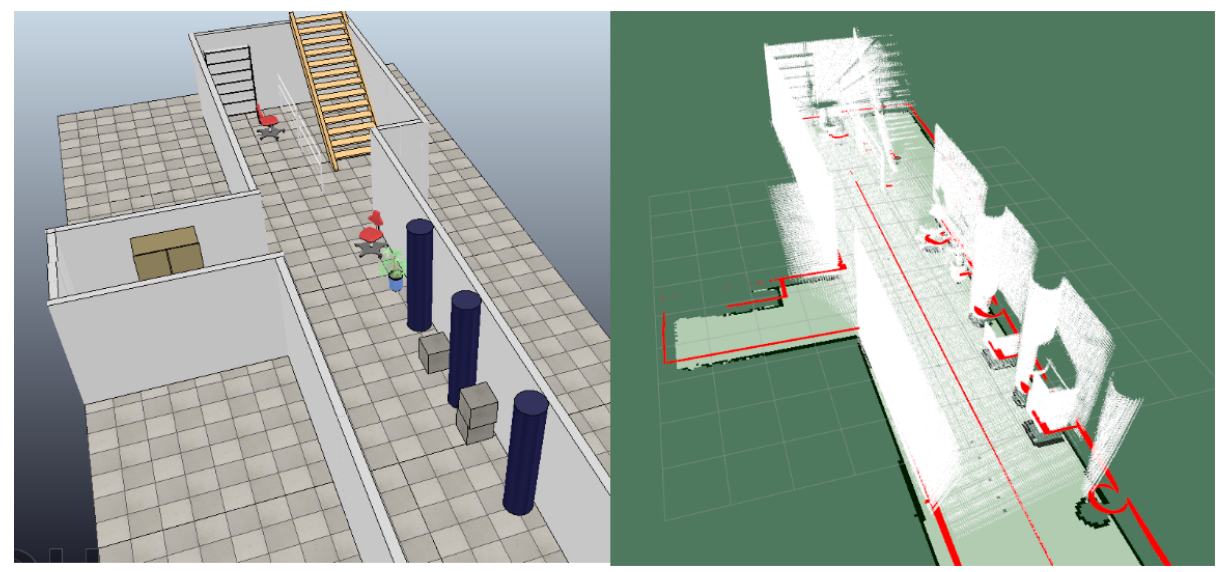

Fig. 5. (left) initial test scene created in VREP (right) Simulated 2D lidar and depth sensor data combined in Rviz. Created by the mobile platform moving in a straight line in a single direction in simulation.

Using this simulation model and data from simulated sensors work has begun on looking at simple user interfaces for teleoperating the platform. This currently has focused on getting the data from the simulated sensors published to ROS so it can be used and displayed elsewhere, and mimics the real system, for this purpose the output from a camera mounted on the front of the platform is shown alongside the map created from Lidar and 3D point cloud data, and the operator controls the platform using standard ROS keyboard teleoperation. An example of the current interface is shown in fig. 6. This was mainly developed to test the integration of the simulation with ROSand is not intended as a final user interface. Using the simulation model different user interfaces can now be developed, using different combinations of simulated sensors. These can then be evaluated and inform the decision on which sensors to implement on the real system, which could result in a significant cost saving as only necessary sensors will need buying.

\section{Conclusion}

A need for reliable flexible robotic systems within the nuclear industry is clear, with the number of facilities requiring decommissioning continuing to rise and current methods being slow, expensive and dangerous. This paper has focused on the plans for the development of a mobile manipulator platform for use in nuclear decommissioning tasks, which is currently in the early stages of development. The platform will combine a mobile base with a manipulator and multiple sensors for visualising the environment. Although the application area considered here is a nuclear one, a flexible mobile manipulator platform could be used in many different areas and so this work could in the future be adapted to other areas. 

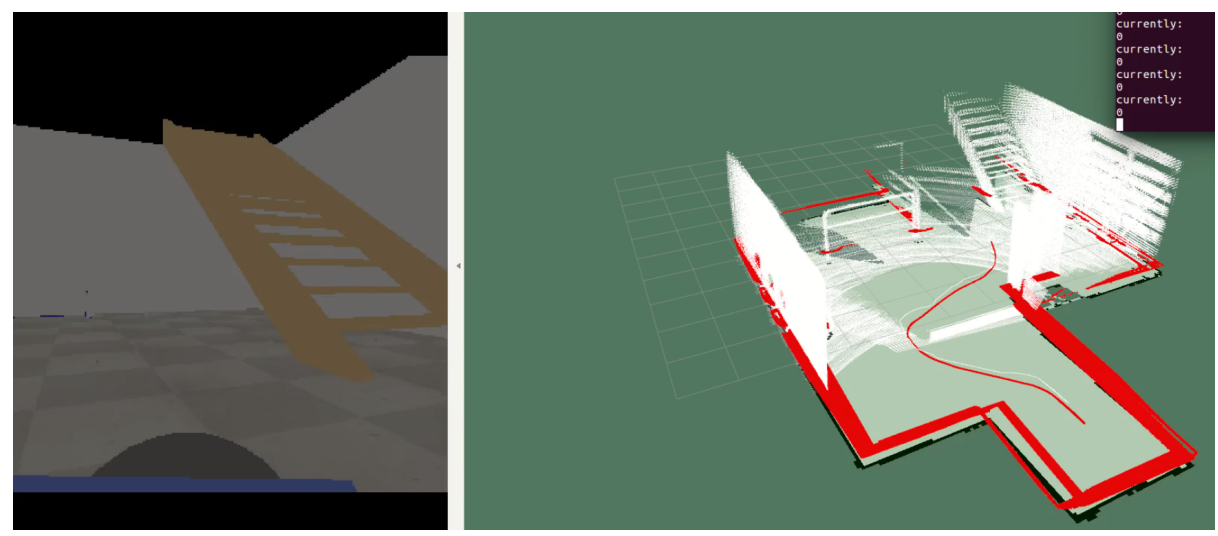

Fig. 6. Snapshot of user interface, showing view from front mounted camera on the left and generated $3 \mathrm{D}$ map to the right.

Details of the simulation model, developed by the present author, are given and some initial results of testing the simulation presented, including a simulated tether and visualisation of combined sensor data. This model will allow testing of different sensor combinations in simulation so the right choice can be made when implemented on hardware, as well as testing of different user interfaces, new control algorithms and novel tether management and path planning systems. There is also potential to use the model for training of new operators as it will share a control interface with the hardware platform, and real environments can be recreated after being mapped in 3D.

Next steps include investigating tele-operation interfaces such as joystick or game-pad controllers and implementing one for the simulation model, this together with the sensor output will allow work on the user interface to begin. At the same time the hardware can be assembled to make the platform and testing can begin, making use of the same algorithms developed for testing the simulation model thanks to both systems using ROS.

\section{References}

1. Bayliss, C., Langley, K.: Nuclear decommissioning, waste management, and environmental site remediation. Elsevier (2003)

2. Brass, P., Vigan, I., Xu, N.: Shortest path planning for a tethered robot. Computational Geometry 48(9), 732-742 (2015)

3. Clearpath Robots: Husky-unmanned ground vehicle. https://www.clearpathrobotics.com/husky-unmanned-ground-vehicle-robot/ (2013), accessed: 2018-12-14

4. E. Rohmer, S. P. N. Singh, M.F.: V-rep: a versatile and scalable robot simulation framework. In: Proc. of The International Conference on Intelligent Robots and Systems (IROS) (2013) 
5. Grisetti, G., Stachniss, C., Burgard, W.: Improved techniques for grid mapping with rao-blackwellized particle filters. IEEE transactions on Robotics 23(1), 34-46 (2007)

6. Guimarães, R.L., de Oliveira, A.S., Fabro, J.A., Becker, T., Brenner, V.A.: ROS navigation: Concepts and tutorial. In: Studies in Computational Intelligence, pp. 121-160. Springer International Publishing (2016)

7. Kim, S., Bhattacharya, S., Kumar, V.: Path planning for a tethered mobile robot. In: 2014 IEEE International Conference on Robotics and Automation (ICRA). IEEE (2014)

8. Kim, S., Likhachev, M.: Path planning for a tethered robot using multi-heuristic a* with topology-based heuristics. In: Intelligent Robots and Systems (IROS), 2015 IEEE/RSJ International Conference on. pp. 4656-4663. IEEE (2015)

9. Nagatani, K., Kiribayashi, S., Okada, Y., Tadokoro, S., Nishimura, T., Yoshida, T., Koyanagi, E., Hada, Y.: Redesign of rescue mobile robot quince. In: 2011 IEEE International Symposium on Safety, Security, and Rescue Robotics. pp. 13-18 (2011)

10. NDA and Innovate UK: Robots compete in nuclear decommissioning challenge. https://www.gov.uk/government/news/robots-compete-in-nucleardecommissioning-challenge (Jan 2018), accessed: 2018-12-10

11. Pitonakova, L., Giuliani, M., Pipe, A., Winfield, A.: Feature and performance comparison of the v-rep, gazebo and argos robot simulators. In: Annual Conference Towards Autonomous Robotic Systems. pp. 357-368. Springer (2018)

12. Quigley, M., Conley, K., Gerkey, B.P., Faust, J., Foote, T., Leibs, J., Wheeler, R., $\mathrm{Ng}$, A.Y.: Ros: an open-source robot operating system. In: ICRA Workshop on Open Source Software (2009)

13. Salzman, O., Halperin, D.: Optimal motion planning for a tethered robot: Efficient preprocessing for fast shortest paths queries. In: 2015 IEEE International Conference on Robotics and Automation (ICRA). IEEE (2015)

14. Sharp, A., Kruusamäe, K., Ebersole, B., Pryor, M.: Semiautonomous dual-arm mobile manipulator system with intuitive supervisory user interfaces. In: Advanced Robotics and its Social Impacts (ARSO), 2017 IEEE Workshop on. pp. 1-6. IEEE (2017)

15. Thrun, S., Burgard, W., Fox, D.: Probabilistic Robotics (Intelligent Robotics and Autonomous Agents series). The MIT Press (2005)

16. Tsitsimpelis, I., Taylor, C.J., Lennox, B., Joyce, M.J.: A review of ground-based robotic systems for the characterization of nuclear environments. Progress in $\mathrm{Nu}-$ clear Energy 111, 109-124 (2019)

17. Universal Robots: Ur5 technical specifications. https://www.universalrobots.com/media/50588/ur5_en.pdf (2014), accessed: 2018-12-14

18. World Nuclear Assosciation: Radioactive waste management (Apr 2018), http://www.world-nuclear.org/information-library/nuclear-fuel-cycle/nuclearwastes/radioactive-waste-management.aspx

19. Xavier, P.: Shortest path planning for a tethered robot or an anchored cable. In: Proceedings 1999 IEEE International Conference on Robotics and Automation (Cat. No.99CH36288C). IEEE (1999)

20. Yoshida, T., Nagatani, K., Tadokoro, S., Nishimura, T., Koyanagi, E.: Improvements to the rescue robot quince toward future indoor surveillance missions in the fukushima daiichi nuclear power plant. In: Yoshida, K., Tadokoro, S. (eds.) Field and Service Robotics: Results of the 8th International Conference. pp. 1932. Springer Berlin Heidelberg, Berlin, Heidelberg (2014) 